\title{
Control for an Autonomous Bicycle
}

\author{
Neil H. Getz \\ Department of Electrical Engineering \\ University of California \\ Berkeley, CA 94720 \\ getz@eecs.berkeley.edu
}

\author{
Jerrold E. Marsden \\ Department of Mathematics \\ University of California \\ Berkeley, CA 94720 \\ marsden(\$math . berkeley . edu
}

\begin{abstract}
The control of nonholonomic and underactuated systems with symmetry is illustrated by the problem of controlling a bicycle. We derive a controller which, using steering and rear-wheel torque, causes a model of a riderless bicycle to recover its balance from a near fall as well as converge to a time parameterized path in the ground plane. Our construction utilizes new results for both the derivation of equations of motion for nonholonomic systems with symmetry, as well as the control of underactuated robotic systems.
\end{abstract}

\section{Introduction}

Control of the bicycle is a rich problem offering a number of considerable challenges of current research interest in the area of mechanics and robot control. The bicycle is an underactuated system, subject to nonholonomic contact constraints associated with the rolling constraints on the front and rear wheels. It is unstable (except under certain combinations of fork geometry and speed) when not controlled. It is also, when considered to traverse flat ground, a system subject to symmetries; its Lagrangian and constraints are invariant with respect to translations and rotations in the ground plane.

Though a number of researchers have studied the stability of bicycles and motorcycles under a nominal linear model of rider control (See Hand [1] for a survey), as far as we know our work presents the first controller allowing tracking of arbitrary trajectories while maintaining balance. Control of balance and roll-angle tracking for the bicycle model we use here has been addressed by Getz [2]. In addition to extending those results to tracking in the plane we also utilize some new results from Bloch, et al. [3] on the derivation of equations of motion for nonholonomic systems with symmetries, and from Getz and Hedrick [4] on the tracking control of nonminimum phase systems.

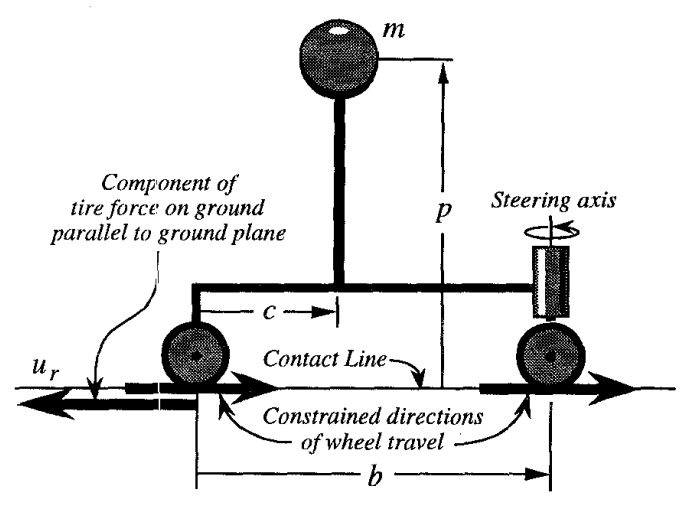

Figure 1: Side view of the bicycle model with $\alpha=0$.

The paper proceeds as follows: By exploitation of the bicycle's constraints and symmetry we first derive a reduced set of equations of motion for the bicycle. We then review how the bicycle can be made to follow arbitrary roll-angle trajectories, thus allowing the vehicle to recover from near falls and disturbances. A purely kinematic model of the bicycle is then introduced in order to show how, disregarding the unstable roll-angle dynamics, the bicycle may be made to converge to a desired trajectory in the plane. We then show how we may use our knowledge of how to steer the kinematic bicycle to construct a controller that allows a leaning bicycle to track planar trajectories without falling.

\section{The Model}

The control of a simplified bicycle model illustrated in Figures 1 and 2 will be considered. The wheels of the bicycle are considered to have negligible inertial moments, mass, radii, and width, and to roll without side or longitudinal-slip. The vehicle is assumed to have a fixed steering-axis that is perpendicular to the flat ground when the bicycle is upright. For simplic- 


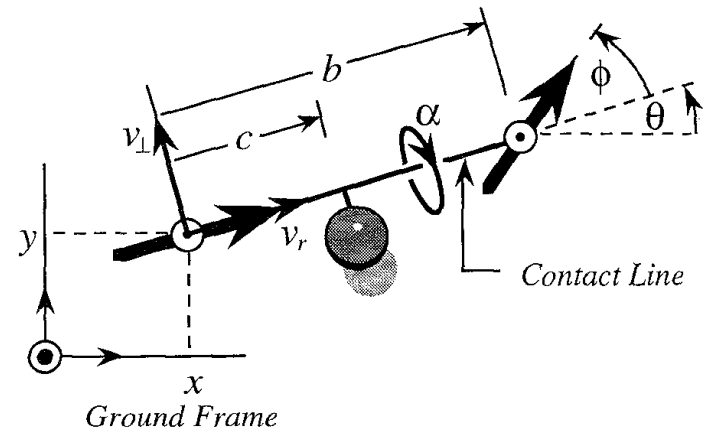

Figure 2: Top view of the bicycle model rolled away from upright by angle $\alpha$. Bold arrows indicate wheel directions at the ground plane.

ity we concern ourselves with a point mass bicycle. The rigid frame of the bicycle will be assumed to be symmetric about a plane containing the rear wheel.

Consider a ground-fixed inertial reference frame with $x$ and $y$ axes in the ground plane and $z$-axis perpendicular to the ground plane in the direction opposite to gravity. The intersection of the vehicle's plane of symmetry with the ground plane forms a contactline. The contact-line is rotated about the $z$-direction by a yaw-angle, $\theta$. The contact-line is considered directed, with its positive direction from the rear to the front of the vehicle. The yaw-angle $\theta$ is zero when the contact-line is in the $x$ direction. The angle that the bicycle's plane of symmetry makes with the ground is the roll-angle, $\alpha \in(-\pi / 2, \pi / 2)$. Front and rear-wheel contacts are constrained to have velocities parallel to the lines of intersection of their respective wheel planes and the ground-plane, but free to turn about an axis through the wheel/ground contact and parallel to the $z$-axis.

Let $\phi \in(-\pi / 2, \pi / 2)$ be the steering-angle between the front-wheel-plane/ground-plane intersection and the contact-line as shown in Figure 2. With $\phi$ we associate a moment of inertia $J$. For simplicity we will parameterize the steering angle by $\sigma:=\tan (\phi / b)$.

The component of the velocity of the rearwheel/ground contact along the contact line is $v_{r}$. The velocity of the rear contact perpendicular to this line and in the ground plane is $v_{\perp}$. The angle of the contact-line with respect to the $x$-axis of the groundfixed inertial frame is $\theta$.

Note that the generalized coordinate corresponding to $v_{r}$ is the the integral in time of the rear-wheel velocity along the path traveled, and the generalized coordinate corresponding to $v_{\perp}$ is the integral in time of the rear-wheel velocity along a direction perpendicular to the rear-wheel which, by virtue of the constraints, is always zero.

It will be assumed that the bicycle exerts a control force $u_{r}$ (see Figure 1) on the ground at the site of the point of contact between the rear-wheel and the ground. The force $u_{r}$ will be considered to act along the contact line as indicated in Figure 1 and is the generalized force corresponding to $v_{r}$. A torque generator is associated with the steering variable $\sigma$, the generalized torque being $u_{\sigma}$. We consider a vehicle with a rigid or non-existent passenger under automatic control.

\section{Equations of Motion}

We choose a body-frame for the bicycle centered at the rear-wheel ground contact, with one axis pointing forward along the line of intersection of the rear wheel plane with the ground, another axis orthogonal to the first and in the ground plane, and an axis normal to the ground, pointing in the direction opposite to gravity (see Figure 2). The body frame is a natural frame in which to write the Lagrangian of the bicycle for a number of reasons. In particular the rolling constraints take on a very simple form. The generalized velocities of the bicycle are contained in the partitioned coordinates $\dot{r}=\left[\dot{\alpha}, v_{r}, \dot{\sigma}\right]^{T}$ and $\dot{s}=\left[\dot{\theta}, v_{\perp}\right]^{T}$. In these velocity coordinates the nonholonomic constraints associated with the front and rear wheels, assumed to roll without slipping, are expressed very simply by $\dot{s}+A(r, s) \dot{r}=0$ or

$$
\left[\begin{array}{c}
\dot{\theta} \\
v_{\perp}
\end{array}\right]+\left[\begin{array}{ccc}
0 & -\sigma & 0 \\
0 & 0 & 0
\end{array}\right]\left[\begin{array}{c}
\dot{\alpha} \\
v_{r} \\
\dot{\sigma}
\end{array}\right]=0
$$

The mapping represented by matrix $A(r, s)$ is an Ehresmann connection [3], connecting the base velocities $\dot{r}$ to the fiber velocities $\dot{s}$. Due to symmetries of the Lagrangian with respect to translations and rotations in the plane, $A(r, s)$ is a function only of $r$.

Let $s_{\alpha}:=\sin (\alpha)$ and $c_{\alpha}:=\cos (\alpha)$. The Lagrangian for the bicycle is

$$
\begin{aligned}
& L=-m g p c_{\alpha}+\frac{1}{2} J\left(\frac{b \dot{\sigma}}{1+b^{2} \sigma^{2}}\right)^{2} \\
& +\frac{1}{2}\left(\left(v_{r}+p s_{\alpha} \dot{\theta}\right)^{2}+\left(v_{\perp}-p \dot{\alpha} c_{\alpha}+c \dot{\theta}\right)^{2}+\left(-p \dot{\alpha} s_{\alpha}\right)^{2}\right)
\end{aligned}
$$

where $m$ is the mass of the bicycle, considered for simplicity to be a point mass, and $J$ is the moment of inertia associated with the steering action.

Incorporating the constraints into the Lagrangian 
we obtain the constrained Lagrangian for the bicycle

$$
\begin{aligned}
& L_{c}=-\left(g m p c_{\alpha}\right)+\frac{b^{2} J \dot{\sigma}^{2}}{2\left(1+b^{2} \sigma^{2}\right)^{2}} \\
& +m\left(\left(v_{r}+p \sigma s_{\alpha} v_{r}\right)^{2}+p^{2} s_{\alpha}^{2} \dot{\alpha}^{2}+\frac{1}{2}\left(c \sigma v_{r}-p c_{\alpha} \dot{\alpha}\right)^{2}\right)
\end{aligned}
$$

Of course the equations of motion for the constrained Lagrangian are not Lagrange's equations. The correct formulation of the equations of motion based upon the constrained Lagrangian are derived in [3] and shown to be equivalent to d'Alembert's equations for constrained systems. They are

$$
\frac{d}{d t} \frac{\partial L_{c}}{\partial \dot{r}^{\alpha}}-\frac{\partial L_{c}}{\partial r^{\alpha}}+A_{\alpha}^{a} \frac{\partial L_{c}}{\partial s^{\alpha}}=-\frac{\partial L}{\partial \dot{s}^{b}} B_{\alpha \beta}^{b} \dot{r}^{\beta}
$$

where $B_{\alpha \beta}^{b}$ denote the coordinates of the curvature of the connection $A(r, s)$,

$$
B_{\alpha \beta}^{b}=\left(\frac{\partial A_{\alpha}^{b}}{\partial r^{\beta}}-\frac{\partial A_{\beta}^{b}}{\partial r^{\alpha}}+A_{\alpha}^{a} \frac{\partial A_{\beta}^{b}}{\partial s^{a}}-A_{\beta}^{a} \frac{\partial A_{\alpha}^{b}}{\partial s^{a}}\right)
$$
form

The reduced equations of motion from $L_{c}$ are of the

$$
M \ddot{r}=F+B u
$$

where $\dot{r}=\left[\dot{\alpha}, v_{r}, \dot{\sigma}\right]^{T}, M \in \mathbb{R}_{3 \times 3}, F \in \mathbb{R}^{3}, B \in \mathbb{R}^{3 \times 2}$, and $u=\left[0, u_{r}, u_{\sigma}\right]^{T}$. The components of $M, F$, and $B$ are

$$
\begin{aligned}
& M_{11}=p^{2}, \quad M_{12}=M_{21}=-c p c_{\alpha} \sigma \\
& M_{13}=M_{31}=M_{23}=M_{32}=0 \\
& M_{22}=1+c^{2} \sigma^{2}+2 p \sigma s_{\alpha}+p^{2} \sigma^{2} s_{\alpha}^{2} \\
& M_{33}=\left(b^{2} J\right) /\left(m\left(1+b^{2} \sigma^{2}\right)^{2}\right) \\
& F^{1}=g p s_{\alpha}+\left(1+p \sigma s_{\alpha}\right) p c_{\alpha} \sigma v_{r}^{2} \\
& +c p c_{\alpha} v_{r} \dot{\sigma} \\
& F^{2}=-\left(1+p \sigma s_{\alpha}\right) 2 p c_{\alpha} \sigma v_{r} \dot{\alpha}-c p \sigma s_{\alpha} \dot{\alpha}^{2} \\
& -\left(c^{2} \sigma+p s_{\alpha}\left(1+p \sigma s_{\alpha}\right)\right) v_{r} \dot{\sigma} \\
& F^{3}=\left(2 b^{4} J \sigma \dot{\sigma}^{2}\right) /\left(m\left(1+b^{2} \sigma^{2}\right)^{3}\right) \\
& B_{11}=B_{12}=B_{22}=B_{3 \downarrow}=0, \quad B_{21}=B_{32}=1 / \mathrm{m}
\end{aligned}
$$

We may further reduce our model through a practical control consideration. We will assume that we have adequate steering torque and a sufficiently small value of the steering inertia $J$ so that we may make $\sigma$ track any smooth trajectory $\sigma(t)$ that we wish. Consequently we will ignore transient behavior of the $\sigma$ and assume that the steering variable will exactly track any signal $\sigma_{d}$ that we wish. This allows us to redefine our controls to be $u_{r}$ and any time derivative of $\sigma$. The variable $\sigma$ is thus decoupled. For convenience later we will choose $\dot{\sigma}$ as a control and call it $w_{\sigma}$. The equations of motion then take on the simpler form

$$
\begin{aligned}
\dot{\sigma} & =w_{\sigma} \\
\tilde{M}\left[\begin{array}{c}
\alpha \\
\dot{v}_{r}
\end{array}\right] & =\tilde{F}+\tilde{B}\left[\begin{array}{c}
u_{r} \\
w_{\sigma}
\end{array}\right]
\end{aligned}
$$

where

$$
\begin{aligned}
\tilde{M} & =\left[\begin{array}{cc}
p^{2} & -c p c_{\alpha} \sigma \\
-c p c_{\alpha} \sigma & 1+\left(c^{2}+p^{2} s_{\alpha}^{2}\right) \sigma^{2}+2 p \sigma s_{\alpha}
\end{array}\right] \\
\tilde{F} & =\left[\begin{array}{c}
g p s_{\alpha}+\left(1+p \sigma s_{\alpha}\right) p c_{\alpha} \sigma v_{r}^{2} \\
-\left(1+p \sigma s_{\alpha}\right) 2 p c_{\alpha} \sigma v_{r} \dot{\alpha}-c p \sigma s_{\alpha} \dot{\alpha}^{2}
\end{array}\right] \\
\tilde{B} & =\left[\begin{array}{cc}
c p c_{\alpha} v_{r} & 0 \\
-\left(c^{2} \sigma+p s_{\alpha}\left(1+p \sigma s_{\alpha}\right)\right) v_{r} & 1 / m
\end{array}\right]
\end{aligned}
$$

Note that the first column of $\tilde{B}$ has $v_{r}$ as a factor confirming the intuitive notion that if $v_{r}=0$ then the steering action can have no affect on either $\alpha$ or $v_{r}$. Also, as $v_{r}$ gets closer and closer to zero the steering velocity $w_{\sigma}$ must get larger and larger in order to maintain influence over $\alpha$ and $v_{r}$. It is practical then to choose controls such that $v_{r}>v_{\min }>0$.

\section{Roll-Angle Tracking}

Let $\alpha_{d}(t)$ ard $v_{r d}(t)$ be desired trajectories for $\alpha(t)$ and $v_{r}(t)$ that remain within the domain of definition of our model. Consider the control

$$
\left[\begin{array}{l}
u_{r} \\
u_{\sigma}
\end{array}\right]=\tilde{B}^{-1}\left(\tilde{M}\left[\begin{array}{l}
V_{\alpha} \\
V_{\sigma}
\end{array}\right]-\tilde{F}\right)
$$

where

$$
\begin{aligned}
& V_{\alpha}=\ddot{\alpha}_{d}-K_{\alpha 1}\left(\dot{\alpha}-\dot{\alpha}_{d}\right)-K_{\alpha 0}\left(\alpha-\alpha_{d}\right) \\
& V_{r}=\dot{v}_{r}-K_{r 0}\left(v_{r}-v_{r d}\right)
\end{aligned}
$$

and the polynomials $s^{2}+K_{\alpha 1} s+K_{\alpha 0}$ and $s+K_{r 0}$ have roots with real parts less than zero. Substituting (10) into (7) and noting that $\tilde{M}$ is nonsingular, it is apparent that by virtue of our choice of input, $\alpha$ and $v_{r}$ are made to exponentially converge to their desired counterparts $\alpha_{d}$ and $v_{r d}$. The graphs of Figures 3 through 5 show an example, from simulation, of how these controls cause the bicycle to recover from a near fall and track a constant roll-angle trajectory and a constant $v_{r d}$. The resulting circular path is shown in Figure 5.

\section{Controlling the Kinematic Bicycle}

By controlling the roll-angle and rear wheel velocity we have not stabilized motion in the ground plane. If a disturbance were to perturb the bicycle from the 


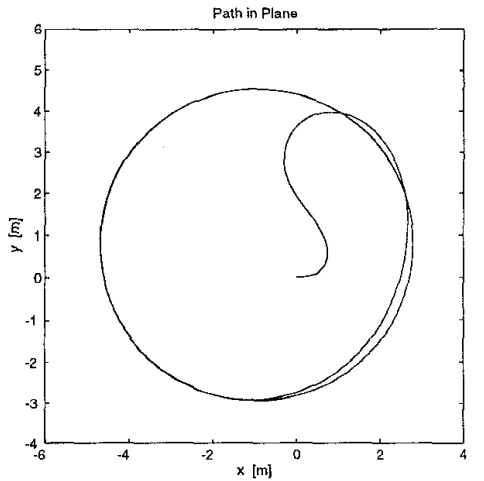

Figure 3: Resulting path in the ground plane for tracking a constant roll angle.
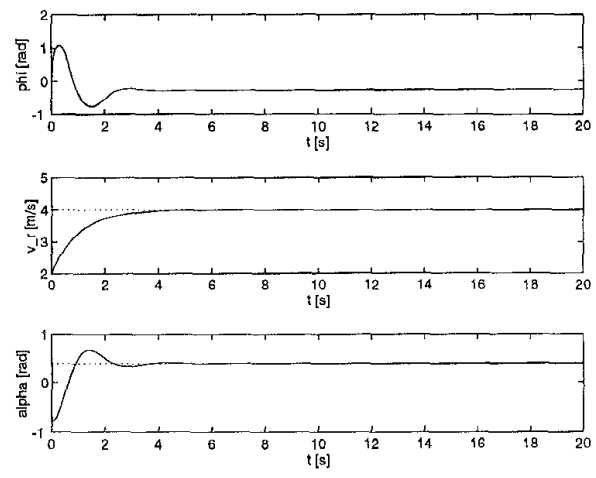

Figure 4: The steering angle $\phi$ (top), rear-wheel velocity $v_{r}$ (middle), and roll angle $\alpha$ (bottom) for tracking of a constant roll angle.

desired roll trajectory, it would quickly recover, but its ground path would no longer be the same circle as before. In this section we will take $x$ and $y$ as our outputs and cause those outputs to track desired counterparts $x_{d}$ and $y_{d}$. We will, for the moment, ignore roll and mass, pretending that the bicycle cannot fall over. We call the resulting model the kinematic bicycle where, as part of the model definition, we maintain

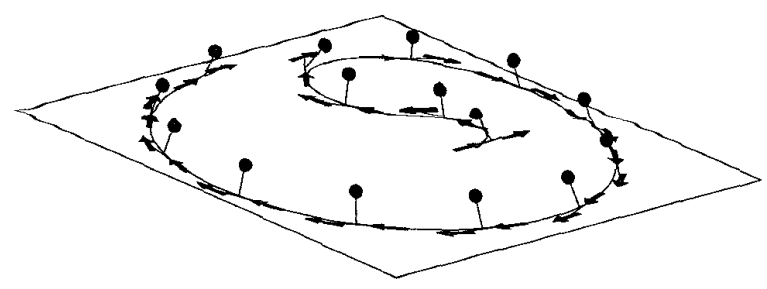

Figure 5: Simulation 1. The first 7 seconds of the ride showing the bicycle's configuration in 0.5 second increments starting from $t=0$. the restriction $v_{r}>v_{\min }>0$.

As derived above the ground plane motion of the bicycle is governed by $\dot{x}=v_{r} c_{\theta}$ and $\dot{y}=v_{r} s_{\theta}$. Differentiating with respect to time gives

$$
\left[\begin{array}{c}
\ddot{x} \\
\ddot{y}
\end{array}\right]=\left[\begin{array}{cc}
c_{\theta} & -v_{r}^{2} s_{\theta} \\
s_{\theta} & v_{r}^{2} c_{\theta}
\end{array}\right]\left[\begin{array}{c}
\dot{v}_{r} \\
\sigma
\end{array}\right]=: G_{x y}\left[\begin{array}{c}
\dot{v}_{r} \\
\sigma
\end{array}\right]
$$

where we have used the fact that $\dot{\theta}=v_{r} \sigma$. Since we have already shown how we can make $v_{r}$ and $\sigma$ be what we want them to be we could use (13) to control $x$ and $y$ along $x_{d}$ and $y_{d}$ as long as $v_{r}>v_{\min }$. Let $V_{x}=\ddot{x}_{d}-K_{x y 1}\left(\dot{x}-\dot{x}_{d}\right)-K_{x y 0}\left(x-x_{d}\right)$ and $V_{y}=$ $\ddot{y}_{d}-K_{x y 1}\left(\dot{y}-\dot{y}_{d}\right)-K_{x y 0}\left(y-y_{d}\right)$. Setting $\ddot{x}=V_{x}$ and $\ddot{y}=V_{y}$ in (13), solving for $\dot{v}_{r}$ and $\sigma$, and calling the results $v_{r d}$ and $\sigma_{d}$ gives

$$
\left[\begin{array}{c}
\dot{v}_{r d} \\
\sigma_{d}
\end{array}\right]=\left[\begin{array}{cc}
c_{\theta} & s_{\theta} \\
-\frac{s_{\theta}}{v_{r}^{2}} & \frac{c_{\theta}}{v_{r}^{2}}
\end{array}\right]\left[\begin{array}{l}
V_{x} \\
V_{y}
\end{array}\right]
$$

Thus if $\dot{v}_{r}=\dot{v}_{r d}$ and $\sigma=\sigma_{d}$, then $x$ and $y$ converge to $x_{d}$ and $y_{d}$ (assuming $\left.v_{r}>v_{\min }\right)^{1}$.

\section{Path Tracking with Balance}

Having determined desired values of $\dot{v}_{r}$ and $\sigma$ which, in the absence of roll-dynamics, would cause the bike to track $\left(x_{d}, y_{d}\right)$, we now seek a bounded trajectory for $\alpha$ compatible with $\dot{v}_{r d}$ and $\sigma_{d}$ (14). We know from Section 4 that we can make $\alpha$ track a smooth trajectory and we know from Section 5 that if we ignore the roll angle, we can make the bicycle follow trajectories in the plane. However, if we ignore roll angle in controlling the trajectory of the bicycle in the plane, the bicycle will fall. Our approach will be to determine a roll angle trajectory that will be compatible with the error dynamics of the ground plane tracking. We will control $\alpha$ to track that angle.

We intend to control $\alpha$ on a much faster time scale than we intend to control the $x, y$ position. This allows us to consider $\sigma_{d}$ and $\dot{v}_{r d}$ to be approximately fixed in the $\alpha$ time scale. We will control $\alpha$ to an "equilibrium" compatible with $\sigma_{d}$ and $\dot{v}_{r d}$. This "equilibrium" is time-varying, depending on the desired path in the plane, as well as the error dynamics associated with following that path. Let $w_{\sigma d}$ denote the value of $w_{\sigma}$ that holds $\sigma$ at $\sigma_{d}$. Recall that $\dot{\sigma}=w_{\sigma}$. The "equilibrium" equation for $\alpha$ is

$$
\begin{aligned}
0 & =g s_{\alpha}+c_{\alpha} \sigma v_{r}+p c_{\alpha} \sigma s_{\alpha} v_{r}+c c_{\alpha} \sigma \dot{v}_{r d}+c c_{\alpha} v_{r} w_{\sigma d} \\
& =F_{\alpha}\left(\alpha, \sigma, v_{r}, \dot{v}_{r d}, w_{\sigma d}\right)
\end{aligned}
$$

\footnotetext{
${ }^{1}$ It is a simple matter to modify the control for the kinematic bike in order to enforce the minimum speed requirement. For simplicity of exposition, however, we do not do this here.
} 
and we will call the solution $\alpha_{*}$. We will show how to obtain a running estimate for $\alpha_{*}$ below. For now assume that we have such an estimate. Define $\hat{F}:=$ $G_{x y}\left[\dot{v}_{r d}, \sigma_{d}\right]^{T}$. Then $\hat{F}$ is the vector field corresponding to the desired motion of $x$ and $y$. The angle $\alpha_{*}$ is a function of the variables $x, y, x_{d}, y_{d}$ and their time derivatives through the dependence of $\sigma_{d}$ and $\dot{v}_{r d}$ on those variables. Thus the Lie derivative ${ }^{2}$ of $\alpha_{*}$ in the direction of $\hat{F}, L_{\hat{F}} \alpha_{*}$ is well defined as is $L_{\hat{F}}^{2} \alpha_{*}$. When $\alpha$ is close to $\alpha_{*}$ and $(x, y)$ is approximately tracking $\left(x_{d}, y_{d}\right)$ then $L_{\hat{F}} \alpha_{*}$ and $L_{\hat{F}}^{2} \alpha_{*}$ are close to $\dot{\alpha}_{*}$ and $\ddot{\alpha}_{*}$ respectively.

We now choose an input $w_{\sigma}$ that causes $\alpha$ to track $\alpha_{*}$. It is

$$
w_{\sigma}=\frac{p V_{\alpha}}{g s_{\alpha}+c_{\alpha} \sigma v_{r}+p c_{\alpha} \sigma s_{\alpha} v_{r}+c c_{\alpha} \sigma \dot{v}_{r}}
$$

where

$$
V_{\alpha}=L_{\hat{F}}^{2} \alpha_{*}-K_{\alpha 1}\left(\dot{\alpha}-L_{\hat{F}}^{2} \alpha_{*}\right)-K_{\alpha 0}\left(\alpha-\alpha_{*}\right)
$$

This may be verified by substituting $w_{\sigma}$ into the reduced equations of motion for $\alpha$. For initial values of $\alpha$ and $\dot{\alpha}$ sufficiently close to $\alpha_{*}$ and $L_{\hat{F}} \alpha_{*}$, and for a choice of $K_{\alpha 1}$ and $K_{\alpha 0}$ with the real parts of the roots of $s^{2}+K_{\alpha 1} s+K_{\alpha 0}$ sufficiently negative, $(\alpha, x, y)$ converges to an arbitrarily small neighborhood of $\left(\alpha_{*}, x_{d}, y_{d}\right)$ exponentially.

We now need only construct an approximation of $\alpha_{*}$. We will construct a dynamical system whose state is the estimator $\hat{\alpha}$,

$$
\dot{\hat{\alpha}}=-\mu F_{\alpha}\left(\hat{\alpha}, \sigma, v_{r}, \dot{v}_{r d}, w_{\sigma d}\right)+\left.L_{\hat{F}} \alpha_{*}\right|_{\alpha=\hat{\alpha}}
$$

where $\mu$ is sufficiently large to consider all arguments of $\hat{F}$ other than $\hat{\alpha}$ approximately fixed. This provides our fastest time scale. Since $\mu \partial \hat{F} / \partial \hat{\alpha}$ is positive in the neighborhood of $\alpha_{*}$ we are guaranteed that $\hat{\alpha}$ converges to a small neighborhood of $\alpha_{*}$ exponentially at a rate determined by $\mu$. The generalization of this "dynamic inversion" of $F$ is presented in Getz and Marsden [5].

Choosing $w_{\sigma}$ according to (16), along with choices of control constants as specified above results in stable approximate tracking of ground plane trajectories while retaining balance. In order to make the controller more robust we may modify $u_{r}$ slightly to assure ourselves that $v_{r}$ remains above $v_{\min }$.

\section{Simulations}

In this section we show the results of simulations of the controlled bicycle converging to and following a straight path and a sinusoidal path.

\footnotetext{
${ }^{2}$ The Lie derivative of a function $\eta: \mathbb{R}^{n} \rightarrow \mathbb{R}$ along a vector field $F$ is defined as $L_{F} \eta=d \eta \cdot F$.
}

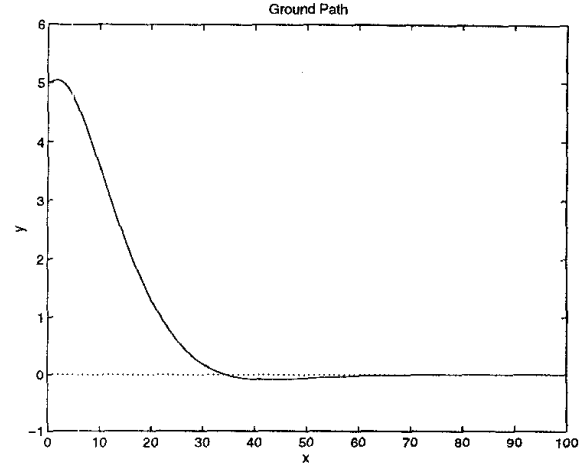

Figure 6: The ground path of the controlled bicycle showing convergence to the target trajectory $x_{d}=$ $10 t[\mathrm{~m}], y_{d}=0$. The scale is in meters.

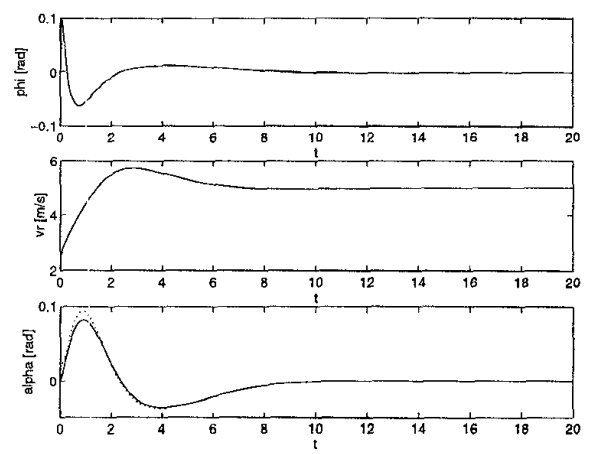

Figure 7: The steering angle (top), rear-wheel velocity $v_{r}$ (middle), and roll angle $\alpha$ (bottom) for the lanechange. The dotted curve is $\alpha_{*}$.

Figures 6 and 7 show the results of a typical lanechange maneuver, where $x_{d}=5 t \mathrm{~m}, y_{d}=0$. The initial conditions of the bicycle were set to be $x(0)=0$, $y(0)=5[\mathrm{~m}], \dot{x}(0)=2.5[\mathrm{~m} / \mathrm{s}], \dot{y}(0)=0, \sigma(0)=0$, $\theta(0)=0, a(0)=0$. The bicycle parameters were $m=30[\mathrm{~kg}], c=1 / 2[\mathrm{~m}], p=1[\mathrm{~m}], b=1[\mathrm{~m}]$, and $g=9.8[\mathrm{~m} / \mathrm{s}]$.

Figure 6 shows the resulting path in the ground plane. Figure 7 shows the steering angle (top), the rear-wheel velocity $v_{r}$ (middle), and the roll angle $\alpha$ (bottom). The path of the rear-wheel contact may be seen to first turn out, then in toward the target. This ground path motion is the result of countersteering, the turning of the front wheel in the direction opposite the direction one wishes to go at the start of a turn. Countersteering can be seen at the far left of the top graph of Figure 7. Note that countersteering comes naturally out of the controller, as it must for a stable turn. Note also how the bicycle rolls in mov- 


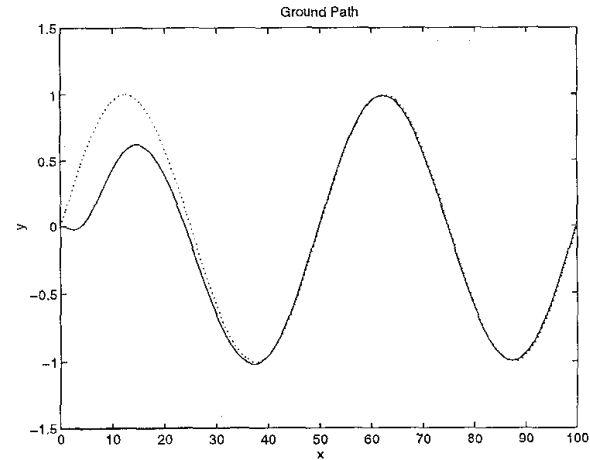

Figure 8: Tracking a sinusoidal path in the plane. Note that $x$ and $y$ are plotted at different scales. The dotted path is the target trajectory.
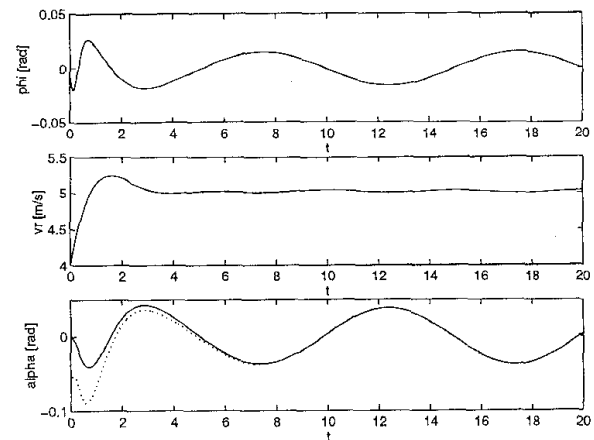

Figure 9: Steering angle $\phi$ (top), rear-wheel velocity $v_{r}$ (middle), and roll-angle $\alpha$ bottom for tracking a sinusoidal path. The dotted curve is $\alpha_{*}$.

ing to the target. The bicycle starts upright, leans right first, then leans left, then straightens again as the target is aprehended. The rear-wheel velocity can be seen to start from its initial value of 2.5 meters per second, move up past the target speed of 5 meters per second, then fall to the target speed as the target is apprehended.

Figures 8 and 9 show the bicycle tracking a sinusoidal trajectory in the ground plane. The initial conditions for the bicycle in this simulation were $v_{r}(0)=4 m / s, \sigma(0)=\alpha(0) \doteq \dot{\alpha}(0)=\theta(0)=x(0)=$ $y(0)=0$. The target trajectory was $x_{d}=5 \mathrm{tm} / \mathrm{s}$ $y_{d}(t)=2 \sin (0.2 \pi t)$. Figure 8 shows the resulting ground-plane path of both the target (dotted) and the bicycle (solid). Again, countersteering is evident in the top graph of Figure 9.

\section{Discussion}

For desired trajectories with large time derivatives larger control gains must be used in order to attain good tracking. The practicality of the controller must be judged on the realizability of such gains. Choice of desired trajectory must also take into account the limitations of the model and the fact that the nonholonomic constraints are only an approximation to the actual tire/road interaction.

\section{Conclusions}

Control of the bicycle is complicated by the nonholonomic constraints on the vehicle as well as the need to track a path in the plane while maintaining balance. We have used the nonholonomic nature of the bicycle to our advantage in obtaining reduced equations of motion. A desired vector field for the ground-plane tracking dynamics was then derived. Using this vector field an equilibrium roll-angle manifold may be calculated dynamically in continuous time. Input/output linearization of the dynamics from steering control to roll angle was then used to stabilize the roll angle to the equilibrium manifold resulting in good tracking behavior with good balance.

The authors are grateful to C.A. Desoer for his comments and advice.

\section{References}

[1] R. S. Hand, "Comparisons and stability analysis of linearized equations of motion for a basic bicycle model," Master's thesis, Cornell, 1988.

[2] N. H. Getz, "Control of balance for a nonlinear nonholonomic non-minimum phase model of a bicycle," in American Control Conference, (Baltimore), American Automatic Control Council June 1994.

[3] A. Bloch, P. Krishnaprasad, J. Marsden, and R. Murray, "Nonholonomic mechanical systems with symmetry," Spring, 1992.

[4] N. H. Getz and J. K. Hedrick, "Constructive regulation and approximate tracking for non-minimum phase systems," in 1995 American Control Conference, (Seattle), American Automatic Control Council, June 1995.

[5] N. H. Getz and J. E. Marsden, "Dynamic inversion of nonlinear maps," Tech. Rep. 621, Center for Pure and Applied Mathematics, Berkeley, California, December 191994. 